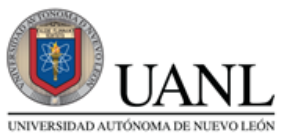

FACPYA
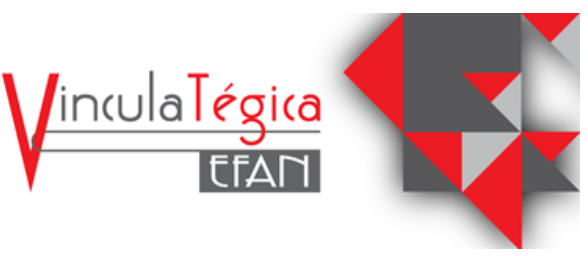

\title{
Control interno y su relación con el desempeño comercial
}

\author{
Humberto Comendeiro ${ }^{1}$ \\ ${ }^{1}$ Universidad Autónoma de Nuevo León, Facultad de Contaduría Pública y Administración, Av. Universidad \\ s/n, col. Ciudad Universitaria, Monterrey, Nuevo León, México, hcomendeirobg@uanl.edu.mx, (+52) 81
}

2943 4045)

\author{
Información del artículo revisado por pares \\ Fecha de aceptación: junio-2021 \\ Fecha de publicación en línea: diciembre-2021 \\ DOI: https://doi.org/10.29105/vtga7.2-63
}

\section{Resumen}

El control interno ha sido reconocido como un instrumento que permite a cualquier organización obtener una seguridad razonable en el cumplimiento de sus objetivos de negocio e informar sobre su gestión a las partes interesadas en ella. Al mismo tiempo, uno de los procesos claves para alcanzar resultados empresariales satisfactorios y sostenibles a largo plazo son las ventas y en un sentido más amplio el desempeño comercial. Con respecto a esto, la evaluación debe quedar restringida a los factores que están bajo control exclusivo de la organización, entre los que se encuentra la gestión del sistema de control interno. Todo ello nos lleva a las conclusiones de que la correcta implementación de los mecanismos de control interno reforzará las áreas comerciales vinculadas al ciclo de Ventas.

Palabras clave: desempeño comercial; control interno; productividad; COSO.

\section{NTRODUCCIÓN}

En la sociedad moderna la globalización se ha convertido en un hecho evidente, entendiéndola como un proceso histórico de convergencia global en los campos de la economía, la política, las tecnologías, así como sociales y culturales, que consiste en una mayor interrelación entre los distintos países del mundo, uniéndolos a través de una serie de cambios sociales y políticos con un carácter global (Andrea Imaginario, s/f).

\begin{abstract}
Internal control has been recognized as an instrument that allows any organization to obtain reasonable assurance in the fulfillment of its business objectives and to inform its stakeholders about its management. At the same time, one of the key processes for achieving successful and sustainable business results in the long term is sales and, more broadly, business performance. In this regard, the evaluation should be restricted to the factors that are under the exclusive control of the organization, among which is the management of the internal control system. All this leads us to the conclusions that the correct implementation of the internal control mechanisms will reinforce the commercial areas linked to the Sales cycle.
\end{abstract}

Keywords: business performance; internal control; productivity; COSO.

Asimismo, la integración paulatina de las fronteras económicas y comunicacionales ha generado una expansión capitalista. Esto, al mismo tiempo, ha favorecido la entrada de capitales y un mayor flujo de transacciones financieras globales orientadas a mercados remotos o emergentes, en condiciones que en el pasado eran muy complicadas, costosas o no viables. La globalización ha cambiado la manera en que se relacionan países e individuos, produciendo un significativo 
impacto en cuestiones económicas como el mercado laboral y el comercio mundial.

En este escenario económico internacional, las empresas púbicas que pretendan ser competitivas necesitan centrar su atención en el uso eficiente y eficaz de sus recursos, ya que, en caso contrario, sus posibilidades de permanecer en el mercado estarían en riesgo.

Para ello es esencial disponer de un sólido sistema de control interno que contribuya al logro de los objetivos propuestos, siendo más relevante para las empresas públicas al brindar confianza y seguridad a inversionistas, accionistas y otras partes interesadas, ya que el control interno es tan importante como un adecuado desempeño económico-financiero, y por otra parte la ausencia o mala implementación de este podría generar dificultades en procesos de índole administrativa, fiscal, contable y financiera.

Las normas y reglamentaciones de tipo fiscal, laboral, ambiental, contable, financiera, societarias, bursátiles o provenientes de organismos federales, estatales y municipales, exigen a las empresas estar al pendiente de los riesgos que el no cumplimiento de estas representa a su patrimonio. A ello se adiciona la necesidad verificar el cumplimiento de las políticas internas de seguridad y control, así como corroborar el acatamiento por las diversas áreas o sectores a las políticas de la empresa.

Por lo anterior, el control interno debe considerarse un elemento clave para la sostenibilidad y crecimiento de las empresas, atendiendo siempre al principio de costobeneficio en su implementación (Hernandez, 2016).

En México, el crecimiento económico y la evolución de los mercados a partir de 1990, en especial el proceso de liberalización que vivió la economía mexicana a inicios de los noventa y la implementación del Tratado de Libre Comercio de América del Norte (TLCAN) en 1994, en un contexto en el que se han llevado a cabo un grupo de transformaciones con el propósito también de liberalizar el comercio internacional y la inversión extranjera directa, propiciar una política monetaria independiente y lograr una adecuada disciplina fiscal (Banco de México, 2018), ha provocado un mayor interés de las empresas nacionales hacia la búsqueda de altos niveles de productividad $y$ competitividad, mediante la aplicación de procedimientos y estándares internacionales, como son el gobierno corporativo y las mejores prácticas centradas en el control interno.

\section{MARCO TEÓRICO}

\subsection{Control interno}

El auge del comercio y las finanzas alcanzado en las ciudades-estado italianas durante el siglo XV, último de la Edad Media, dio lugar a una transformación de la contabilidad como técnica de asiento de las transacciones, surgiendo la teneduría de libros para registrar las operaciones de los negocios.

En ese entorno mercantil, el matemático y fraile franciscano Fray Lucas Pacioli, precursor del cálculo de probabilidades y la contabilidad moderna, publicó en 1494 su libro "La suma de aritmética, geometría, proporciones y proporcionalidad", donde analiza el uso de la partida doble por los contadores venecianos, documentando este método y añadiendo elementos para perfeccionarlo, razonando el hecho económico desde dos puntos de vista: partida y contrapartida. Se considera que este suceso marca el origen del control interno.

Sin embargo, no fue hasta mediados del siglo XIX que, como resultado de la Revolución Industrial iniciada en el Reino de Gran Bretaña y el conjunto de transformaciones económicas, tecnológicas y sociales que ello provocó, aparece la necesidad de un mayor control sobre las operaciones y procesos cada vez más complejos.

De acuerdo con Rivero y Campos (2010), en esta etapa los dueños de los negocios no pudieron continuar atendiendo directamente las cuestiones productivas, comerciales y administrativas, viéndose en la necesidad de traspasar funciones dentro de la organización, al mismo tiempo que se desarrollaron los primeros sistemas y procedimientos para prevenir o reducir los 
fraudes y/o errores. Así aparece el control como una función administrativa, para garantizar el cumplimiento de los objetivos y políticas preestablecidas.

Derivado de lo anterior, los contadores concibieron la comprobación interna, que para Dicksee (1905) incluía tres elementos, como son la división de funciones, utilización de los registros de contabilidad y rotación del personal. El término fue posteriormente reemplazado por el de control interno.

A mediados de la década de 1980 en los EE. UU., el entorno marcado por la desregularización impulsada por la Administración Reagan, la subida sin precedentes de las tasas de interés por parte del Sistema de la Reserva Federal, la crisis generalizada del sistema bancario y las prácticas fraudulentas de varias cajas de ahorro, produjo la mayor quiebra de instituciones financieras desde la Gran Depresión, siendo denominada como "Crisis de ahorros y préstamos" (Moysich, 1997), generando un grave problema de confianza en la sociedad norteamericana que extendió su impacto negativo a la economía y los mercados, lo que llevó al Congreso de ese país a tratar de identificar las posibles causas, poniendo énfasis en si estos hechos pudieron haberse evitado de existir mejores prácticas de auditoría (Grundfest \& Berueffy, 1989).

En este contexto de escrutinio público, una respuesta del sector privado a estas acciones legislativas llegó de la mano de cinco de las más importantes asociaciones de contadores públicos en EE. UU, que decidieron patrocinar conjuntamente a la Comisión Nacional sobre Información Financiera Fraudulenta, también conocida como "Comisión Treadway", en la elaboración de un estudio para tratar de ubicar las causas raíces que podrían dar espacio a la emisión de información financiera fraudulenta y ofrecer medidas alternativas para disminuir sus consecuencias (COSO, s/f).

Los estudios de la Comisión se extendieron por espacio de dos años y el informe final se emitió a finales de 1987 con recomendaciones enfocadas mayormente a las empresas públicas, los auditores externos, la Comisión de Bolsa y Valores de Estados Unidos (SEC por sus siglas en inglés) y otros organismos con facultad para intervenir sobre el entorno legal o regulatorio y la comunidad académica (COSO, s/f).

Al final de sus propuestas a las empresas públicas, el informe Treadway aconsejaba que sus organizaciones patrocinadoras prosiguiesen trabajando en conjunto para sintetizar los diferentes conceptos y definiciones de control interno y constituir una referencia común (Treadway Jr. et al., 1987, p. 48).

A la vista de esta observación, las organizaciones patrocinadoras constituyeron el Comité de Organizaciones Patrocinadoras de la Comisión Treadway (COSO), una organización privada que tiene como misión "proporcionar liderazgo intelectual a través del desarrollo de marcos y orientación sobre gestión de riesgos empresariales, control interno y disuasión del fraude" (COSO, s/f).

Como resultado de los esfuerzos de COSO en ahondar en la elaboración de una noción común sobre el control interno, en 1992 aparece la publicación "Control Interno: Marco Integrado" también conocido como "Informe COSO".

En 2004, COSO realizó una nueva publicación del documento llamado "Marco Integrado de Gestión de Riesgos Empresariales", también llamado "COSOERM" que entrega un enfoque más sólido y amplio sobre la identificación, evaluación y gestión del riesgo en las organizaciones. Del mismo modo, en mayo de 2013 publicó la versión actualizada del "Marco Integrado de Control Interno". Por último, en 2017 se divulgó otra versión actualizada de COSO en un documento titulado "Gestión de Riesgo Empresarial-integrando Estrategia y Desempeño".

En el Marco Integrado de Control Interno vigente diseñado por COSO, se establecen tres tipos de objetivos que ayudan a las organizaciones a enfocarse en distintos aspectos del control interno: operativos, de información y de cumplimiento.

Al mismo tiempo, el sistema de control interno está segmentado en cinco componentes integrados que se enlazan con 
los objetivos de la empresa: entorno de control, evaluación de los riesgos, actividades de control, sistemas de información y comunicación, y actividades de monitoreo y supervisión.

Existe un vínculo directo entre los objetivos de la organización, los componentes y la estructura administrativa.

Así mismo, dentro de cada componente se introducen 17 principios que representan los criterios esenciales y son aplicables a los objetivos antes descritos. Los principios permiten estimar la efectividad del sistema de control interno.

Todas estas contribuciones han robustecido el marco conceptual de COSO, dotándole de una mayor fortaleza y trascendencia.

De este modo, con el transcurso del tiempo el modelo COSO y las propuestas de la Comisión Treadway fueron recibiendo atención en EE. UU. entre las grandes empresas que cotizan en bolsa y gradualmente se fue extendiendo a otros países, de tal forma que se convirtió en un nuevo paradigma de control interno a nivel global presente en todo tipo de entidades. Esta evolución llegó a su punto máximo con la promulgación 2002 de la Ley de Protección al Inversionista y Reforma Contable de Empresas Públicas de 2002, también conocida como Ley Sarbanes-Oxley o ley SOx.

Eventualmente, otras instituciones profesionales han adoptado el modelo COSO como referencia para sus propias investigaciones y desarrollos metodológicos. Entre las más prominentes, por su alcance internacional, se encuentran la Federación Internacional de Contadores (IFAC) que ha introducido el modelo COSO a sus normas de auditoría (IFAC, 2012, p. 21) y la Organización Internacional de Entidades Fiscalizadoras Superiores (INTOSAI), cuyas normas de control interno para el sector público se basan en el modelo citado (INTOSAI, 2004). El Informe COSO también fue adoptado por el Banco Mundial (Choudhury \& Mitchell, 2000) y otros organismos financieros a través del mundo.
Es preciso mencionar que después de la aparición del modelo COSO, se han publicado otros modelos de control en diversos países, entre los que han gozado de más difusión internacional destacan:

— "Informe King sobre Gobierno Corporativo". Se publicaron informes en 1994 (King I), 2002 (King II) y 2009 (King III) y una cuarta revisión (King IV) en 2016. El acatamiento del informe King es una condición necesaria para las empresas cotizadas en la Bolsa de Valores de Johannesburgo.

- "Criterios de la guía de control de la Junta de Control" (COCO): Este modelo fue dado a conocer en 1995 por el Instituto Canadiense de Contadores Certificados (CICA).

- "Código de Gobierno Corporativo del Reino Unido": Según las reglas de cotización de la Autoridad de Conducta Financiera (FCA), todas las empresas con una cotización premium de acciones de capital en el Reino Unido deben revelar en su informe anual sobre cómo han aplicado el código, bajo el enfoque de "cumplir o explicar". Los orígenes del documento se derivan del "Informe Cadbury" e "Informe Greenbury" publicados en 1992 y 1995 respectivamente, lo que dio paso en 1998 al "Código Combinado". Por otra parte, para las empresas que aplican estos lineamientos, la guía con respecto al control interno es el "Informe Turnbull" de 1999.

Por sobre sus diferencias, todos estos modelos recalcan que el control interno de las organizaciones es responsabilidad de los órganos de gobierno y de la alta dirección, como vía de alcanzar una seguridad razonable sobre el cumplimiento de los objetivos de la organización, para lo cual se apoyan de los comités de auditoría y la auditoría interna. En este contexto, el modelo COSO continúa siendo una referencia legítima para explicar las nuevas tendencias del control interno, inclusive en las áreas de competencia donde el modelo no es totalmente aplicable (Miaja, 
2010).

En el caso de México, de acuerdo con Serrano (2016), tanto la Secretaría de la Función Pública como la Auditoría Superior de la Federación tienen como punto de referencia a las orientaciones de COSO, por lo que se podría asumir que ya sea en el Gobierno y las entidades federativas como en el ámbito de las empresas públicas de México, es COSO el marco que más se ajusta a sus necesidades y características, permitiendo disponer de sistemas de control interno que ayuden a contar con lineamientos para una mejor gestión y dirigir esfuerzos para crear valor en los procesos que se lleven a cabo.

\subsection{Desempeño comercial}

Para Primo (2017) el desempeño comercial es de gran importancia para alcanzar resultados empresariales satisfactorios y sostenibles a largo plazo, pues de esto depende la captación de clientes y la realización de las ventas e ingresos. En una época de rápidos avances en las tecnologías de la información, el entorno para las empresas públicas es cada vez más competitivo y dinámico, razón por la que la función comercial puede ser un componente esencial para mejorar la eficacia en ventas y la diferenciación competitiva.

De acuerdo con Castro (2014), el área comercial actúa también como enlace entre la empresa y el mundo exterior, razón por la que es imprescindible disponer de un sistema de control interno que aplicado a los procesos que contribuyen a la productividad, permita evaluar permanentemente el desempeño y suscitar ciclos de mejora continua, repercutiendo en el incremento de las ventas, la participación del mercado y clientes más satisfechos.

Para Bendle et al (2016), los costos de la fuerza de ventas representan el 10 por ciento o más de los presupuestos operativos de una gran cantidad de empresas públicas. $\mathrm{Su}$ eficacia es fundamental para las valoraciones del mercado de valores, que a menudo se basan en supuestos agresivos para la adquisición de clientes y el crecimiento continuo.

Según el Diccionario de Marketing de Lenguaje Común (s/f), mejorar la eficacia de las ventas no es solo un problema de la función de ventas; es un problema de la empresa, ya que requiere una colaboración profunda entre ventas y marketing para comprender qué funciona y qué no, y la mejora constante del conocimiento, los mensajes, las habilidades y las estrategias que el personal de ventas aplica cuando trabaja con oportunidades de ventas.

En ese sentido, en 2002, Baldauf y Cravens contribuyeron al estudio de las variables que rigen el desempeño comercial creando un vínculo entre la efectividad del vendedor $\mathrm{y}$, por otro lado, elementos como el tipo de producto que se comercializa y el ritmo de crecimiento del mercado.

Algunas investigaciones previas también incluyeron el estudio de variables como la estructura de ventas, eficacia y tamaño de la fuerza de ventas y el diseño de los territorios de venta. Sin embargo, muchos de estos trabajos se centraron en investigar las características de los vendedores como presuntos indicadores del desempeño comercial (Primo, 2017).

Una variable poco estudiada en la literatura científica respecto al tema es el efecto que el control interno genera sobre este proceso, ya que al estar bajo la influencia directa de la propia empresa permitiría evaluar el cumplimiento de los objetivos propuestos y conocer las oportunidades de mejora en cada una de las etapas y procedimientos. Entendido así, se puede afirmar que el control interno se convierte en un factor clave del éxito en el desempeño comercial.

Por lo tanto, en primera instancia, es necesario precisar lo que comprende la función comercial. Según refieren Küster y Canales (2008), esta incluye dos variables: efectividad y desempeño comercial. El desempeño es un resultado que se evalúa en términos de su contribución a los objetivos de negocios, mientras que la efectividad se refiere a ciertos indicadores de resultados (por ejemplo: ventas, nuevos clientes, rentabilidad, cuota de mercado, etc.).

Bajo esta concepción, para Munuera y Román (2003), el desempeño depende mayormente de factores que quedan bajo el 
control de la empresa, si bien estos pueden ser modificados por la alta dirección a través de políticas internas y otras decisiones concernientes a la empresa tales como los objetivos, las particularidades de los recursos humanos y financieros, la innovación y los recursos dedicados a la investigación, la labor de mercadotecnia y la calidad del producto. $\mathrm{Y}$, al contrario, la efectividad depende, además de los factores antes mencionados, de otras circunstancias que quedan fuera del control de la empresa. Se trata de cuestiones asociadas al contexto tales como los clientes, la competencia, las características del país o territorio, las limitaciones de curso legal, la tecnología, los recursos naturales, etc.

En conclusión, los resultados generales alcanzados por el área comercial o de ventas están marcados por el entorno, así como por la gestión y características de la empresa. Por consiguiente, tal y como recogen diversos investigadores (Primo, 2017), los resultados no pueden atribuirse únicamente a las actividades realizadas por ésta, sin tomar en consideración factores externos. Con respecto a esto, la evaluación del desempeño comercial debe quedar restringido a los factores que están bajo control exclusivo de la organización, entre los que se encuentra la gestión del sistema de control interno.

\section{MÉTODO}

Este trabajo no implica actividades empíricas, ya que es una investigación descriptiva para delinear los fundamentos teóricos de los resultados que las empresas públicas de Nuevo León, cotizadas en la Bolsa Mexicana de Valores (BMV), podrían tener en la productividad y el desempeño comercial al implementar un modelo de control interno para controlar sus operaciones, tomando como referencia el Marco de Control Interno COSO, considerando la amplia aceptación que este ha tenido a nivel internacional, y especialmente en la comunidad de empresas que cotizan en las distintas bolsas de valores del mundo, incluyendo la mexicana.

\section{HIPÓTESIS}

H1: El empleo y una adecuada cultura de control interno en las empresas regiomontanas que cotizan en la BMV afectan la productividad de las operaciones comerciales y como consecuencia su desempeño.

Ho: El empleo y una adecuada cultura de control interno en las empresas regiomontanas que cotizan en la BMV no afectan la productividad de las operaciones comerciales y por lo tanto, tampoco su desempeño.

\section{RESULTADOS}

Hasta la fecha no está regulado un marco integral de control interno específico para las empresas públicas mexicanas que cotizan en la BMV.

Y aunque el modelo COSO ha tomado gran relevancia debido a la exigencia de aplicar un modelo reconocido de control interno a las empresas públicas que participan en los principales mercados financieros internacionales, se estima que continúa siendo baja su aplicación por parte de la comunidad empresarial nacional.

En materia legal, la Ley del Mercado de Valores (LMV) de 2005 es el principal instrumento al que deben ceñirse las empresas que cotizan en el mercado de valores en México. Sin embargo, no se puede perder de vista la existencia del Código de Mejores Prácticas Corporativas (CMPC) que a iniciativa del Consejo Coordinador Empresarial (CCE) se publicó en 1999; y si bien el Gobierno Corporativo no es un instrumento vinculante para todas las empresas del país, sí es un documento con recomendaciones muy importantes para las empresas públicas.

El objetivo final que buscan tanto la LMV como el CMPC con relación al control interno, es proteger los intereses de los accionistas y los activos de la compañía, es decir, establecer mecanismos para una adecuada "vigilancia" de la sociedad mercantil, con instrumentos acordes a sus necesidades.

No obstante, pareciera que no se ha hecho suficiente énfasis a la comunidad empresarial en utilizar el control interno como una herramienta para potenciar el desempeño comercial en las empresas que 
buscan mejorar su gestión basadas en las mejores prácticas corporativas a nivel internacional.

La afirmación anterior encuentra eco en un análisis realizado por Deloitte (2010), en el que se afirma que entre las razones fundamentales por las que las empresas mexicanas no adoptan sistemas de control interno es porque consideran que conlleva demasiados costos en comparación con los beneficios que representa.

Asimismo, afirma el estudio de Deloitte, que la falta de institucionalización en la creación y crecimiento de las empresas provoca que éstas carezcan tanto de una adecuada proyección estratégica como de cada una de las áreas funcionales de la organización, impidiendo el uso del control interno como vía para conseguir un mayor desempeño comercial y eficacia en ventas, que les permita continuar como negocio en marcha garantizando la realización de los objetivos empresariales.

En ocasiones el sistema de control interno se adopta primero en aquellas organizaciones que comienzan experimentar un crecimiento apreciable y van requiriendo de mejores prácticas de control para salvaguardar los recursos de la empresa y verificar el cumplimiento de la legislación aplicable, cuando debiera ser a la inversa, pues es precisamente el control unas de las variables bajo el control de la propia empresa, que bien instrumentado se convierte un factor decisivo para el éxito corporativo, independientemente del tamaño de la organización.

\section{CONCLUSIONES}

El control interno no es una invención reciente, sino que se remonta a, cuando menos, la Edad Media. Fue introducido conceptualmente por primera vez como un sistema por el inglés Lawrence R. Dicksee a principios del siglo XX.

Ha habido un siglo de debate sobre la definición de controles internos $\mathrm{y}$, por lo general, los cambios a las definiciones existentes han sido controvertidos. Existen diferentes definiciones de control interno, pero el marco de control interno emitido por el COSO en 1992 se ha convertido en el modelo mundial.

Las divulgaciones públicas de los controles internos representan una característica clave de las leyes, regulaciones y códigos de gobierno corporativo en todo el mundo, asumiendo un mayor protagonismo, entre otros factores, por la creciente complejidad de la función comercial en la empresa pública.

A ese respecto, el control interno puede funcionar como un facilitador o como un obstáculo para el desempeño comercial, por lo que un sistema de controles internos poco dinámico o desenfocado podría tener un impacto negativo en el desempeño organizacional y viceversa, afectando potencialmente el estado de ganancias y pérdidas de las empresas.

Aunque en una gran parte de la literatura consultada sobre el tema los controles están asociado con los costos correspondientes, asimismo deben ser asociados con los beneficios, ya que los controles también propician que todo se haga bien. Por tanto, los controles y el riesgo tienen beneficios que pueden estar asociados positivamente con el desempeño de la empresa. Esto está relacionado con los controles internos sobre la efectividad y el desempeño en las operaciones comerciales y la manera en que esos controles brindan seguridad en el cumplimiento de los objetivos de la sociedad mercantil.

Existe la consideración de que controles internos más estrictos proveerán una mayor garantía de que las empresas sean efectivas y tengan un rendimiento favorable en sus operaciones comerciales, es decir, que sean eficientes en cuanto a los recursos y, al mismo tiempo, logren los objetivos establecidos relacionados con el desempeño. Por otra parte, se estima que controles internos más débiles inhabilitarán tal desempeño. Sin embargo, asociar los controles internos con la eficacia y el desempeño en las operaciones de la empresa no está exento de dificultades. Una cuestión importante en este caso es definir adecuadamente el desempeño comercial.

La base de este argumento es que las 
buenas prácticas de control interno pueden ser una fuente de ventaja competitiva, que se traducirían en controles mejor diseñados, mejor equilibrados e integrados, lo que a su vez podría tener un efecto positivo en el desempeño organizacional en general.

Uno de los desafíos que tendrá el empresariado neoleonés en el corto plazo será la comprensión e implementación de un marco de control interno que no solo funcione para supervisar actividades y tareas, sino que también permita la innovación y la creatividad para contribuir al logro de los objetivos del negocio, desde la perspectiva del desempeño comercial. 


\section{REFERENCIAS}

Andrea Imaginario. (s/f). Globalización. Significados.com. https://www.significados.com/globalizacion/

Baldauf, A. and Cravens, D. W. (2002). The effect of moderators on the salesperson behavior performance and salesperson outcome performance and sales organization effectiveness relationships. European Journal of Marketing, 36(11/12), 1367-1388. https://doi.org/https://doi.org/10.1108/03090560210445227

Banco de México. (2018). Informe trimestral julio - septiembre 2018. https://www.banxico.org.mx/publicaciones-y-prensa/informes-trimestrales/\%7B59EC89F815C7-9526-8E46-48B52C302626\%7D.pdf

Bendle, N., Ferris, P., Pfeifer, P., \& Reibstein, D. (2016). Marketing Metrics The Manager's Guide to Measuring Marketing Performance (Third Edit). Pearson Education, Inc. https://www.nima.today/wp-content/uploads/2018/11/Marketing-Metrics-Neil-T.-BendlePaul-W.-Farris-Philip-E.-Pfeifer-And-David-J.-Reibstein.pdf

Castro, J. (2014, diciembre 2). ¿Cómo medir el desempeño de tu proceso comercial? Blog Corponet. https://blog.corponet.com.mx/como-medir-el-desempeno-de-tu-proceso-comercial

Choudhury, F. H., \& Mitchell, P. (2000). COSO in the Worldbank. En S. L. van Biene-Hershey M.E. (Ed.), Integrity and Internal Control in Information Systems (Vol. 37, pp. 1-10). Springer. https://doi.org/10.1007/978-0-387-35501-6_1

Common Language Marketing Dictionary. (s/f). Sales Force Effectiveness. https://marketingdictionary.org/s/sales-force-effectiveness/

COSO. (s/f). History. https://www.coso.org/Pages/aboutus.aspx

Deloitte. (2010). Guías de referencia sobre Control Interno en México (Número Invierno 2010). https://www2.deloitte.com/content/dam/Deloitte/mx/Documents/risk/GobiernoCorporativo/guia-control-interno-mx.pdf

Dicksee, L. R. (1905). Auditing: A Practical Manual for Auditors (R. Montgomery (ed.); 1976a ed.). Arno Press.

Grundfest, J. A., \& Berueffy, M. (1989). The Treadway Comissioin Report: Two years later (Número 202). https://www.sec.gov/news/speech/1989/012689grundfest.pdf

Hernandez, A. (2016). El control interno contable y fiscal como medida para contribuir a la maximizacion de los resultados financieros de los negocios. Innovaciones de Negocios, 13(25), 47-69. http://eprints.uanl.mx/12630/1/13.25 A3.pdf

IFAC. (2012). Evaluar y mejorar el control interno en las organizaciones. Federación Internacional de Contadores. https://www.ifac.org/system/files/publications/files/Evaluar-y-mejorar-elcontrol-interno-en-las-organizaciones.pdf

INTOSAI. (2004). Guía para las normas de control (p. 87). Organización Internacional de Entidades Fiscalizadoras Superiores. https://www.asf.gob.mx/uploads/62_Documentos_tecnicos/Normas_Internacionales.pdf

Küster, I., \& Canales, P. (2008). Some determinants of salesforce effectiveness. Team Performance Management: An International Journal, 14, 296-326. https://doi.org/10.1108/13527590810912304

Miaja, M. (2010). Las tendencias actuales en los sistemas de control interno de las organizaciones . Implicaciones para las Administraciones Públicas. Documentación Administrativa, 286-287, 207-237. https://doi.org/https://doi.org/10.24965/da.v0i286-287.9670

Moysich, A. (1997). The Savings and Loan Crisis and Its Relationship to Banking. En History of the Eighties, Lessons for Future (Vol. 1, pp. 167-188). Federal Deposit Insurance 
Corporation. http://www.fdic.gov/bank/historical/history/vol1.html

Munuera Alemán, J., \& Román Nicolás, S. (2003). Conceptualización y medición del rendimiento y la efectividad de la fuerza de ventas. Esic market, 114, 23-42.

Primo, D. (2017). Propuesta de un modelo explicativo de la efectividad, rendimiento y control estratégico de la red comercial. Una aplicación al sector financiero. [Tesis de doctorado, Universidad Complutense de Madrid]. https://eprints.ucm.es/40771/1/T38228.pdf

Rivero, A., \& Campos, L. (2010). Antecedentes y evolución del control interno, su desarrollo en Cuba. Monografías.com. https://www.monografias.com/trabajos59/evolucion-controlinterno/evolucion-control-interno.shtml

Serrano, J. A. (2016). El control interno de la Administración Pública: ¿elemento de estancamiento o de desarrollo organizacional? D3 Ediciones SA de CV. http://archivos.diputados.gob.mx/Centros_Estudio/UEC/prods/EL CONTROL INTERNO DE LA ADMINISTREACION PUBLICA.pdf

Treadway Jr., J. C., Batten, W. M., Kanaga, W. S., Marsh, H. L., Storrs, T. I., \& Trautlien, D. H. (1987). Report of the National Commission on Fraudulent Financial Reporting. National Commission on Fraudulent Financial Reporting. https://documents.pub/document/ncffr-oct1987.html 